\title{
A HAMILTONIAN APPROXIMATION TO SIMULATE SOLITARY WAVES OF THE KORTEWEG-DE VRIES EQUATION
}

\author{
MINGYOU HUANG
}

\begin{abstract}
Given the Hamiltonian nature and conservation laws of the Korteweg-de Vries equation, the simulation of the solitary waves of this equation by numerical methods should be effected in such a way as to maintain the Hamiltonian nature of the problem. A semidiscrete finite element approximation of Petrov-Galerkin type, proposed by $\mathrm{R}$. Winther, is analyzed here. It is shown that this approximation is a finite Hamiltonian system, and as a consequence, the energy integral

$$
I(u)=\int_{0}^{1}\left(\frac{u_{x}^{2}}{2}+u^{3}\right) d x
$$

is exactly conserved by this method. In addition, there is a discussion of error estimates and superconvergence properties of the method, in which there is no perturbation term but instead a suitable choice of initial data. A single-step fully discrete scheme, and some numerical results, are presented.
\end{abstract}

\section{The Hamiltonian NatUre and CONSERVATION LAWS}

In this paper, we shall consider the following problem for the Kortewegde Vries equation:

$$
\begin{aligned}
& u_{t}-6 u u_{x}+u_{x x x}=0, \quad x \in R, t \geq 0, \\
& u(x+1, t)=u(x, t), \\
& u(x, 0)=u_{0}(x) \quad \text { (a prescribed 1-periodic function). }
\end{aligned}
$$

To study the Hamiltonian nature of problem $(\mathrm{P})$, we introduce the following function space with $I=[0,1]$,

$$
H_{p}^{m}=\left\{v \in H^{m}(I) ; v^{(i)}(x+1)=v^{(i)}(x), i=0,1, \ldots, m-1\right\},
$$

and the functional

$$
H(u)=\int_{0}^{1}\left(\frac{u_{x}^{2}}{2}+u^{3}\right) d x,
$$

Received March 24, 1989; revised December 7, 1989, May 22, 1990.

1980 Mathematics Subject Classification (1985 Revision). Primary 65N15, 65N30.

Key words and phrases. Korteweg-de Vries equation, finite element method, Hamiltonian approximation, superconvergence.

Supported by the National Natural Science Foundation of China and the Volkswagen Foundation of Germany. 
where $u^{(i)}=\partial^{i} u / \partial x^{i}$. Define

$$
\delta / \delta u:=\sum_{k=0}^{\infty}(-1)^{k}(d / d x)^{k} \partial / \partial u^{(k)}
$$

then $\delta H / \delta u=3 u^{2}-u_{x x}$, and problem (P) is equivalent to finding a map $u(t)$ from $\mathbf{R}^{+}$to $H_{p}^{m}$ such that

$$
u_{t}=J \delta H / \delta u, \quad J=\partial / \partial x
$$

Since

$$
\int_{0}^{1} \frac{\delta H}{\delta u} \frac{\partial}{\partial x}\left(\frac{\delta H}{\delta u}\right) d x=0, \quad u \in H_{p}^{m}
$$

then for any solution $u=u(t)$ of $\left(\mathrm{P}^{\prime}\right)$ we have

$$
\frac{d H(u)}{d t}=\int_{0}^{1} \frac{\delta H}{\delta u} \frac{\partial u}{\partial t} d x=\int_{0}^{1} \frac{\delta H}{\delta u} \frac{\partial}{\partial x} \frac{\delta H}{\delta u} d x=0,
$$

i.e., $u=u(t)$ satisfies the energy conservation law: $H(u(t))=$ const.

For any functionals $T$ and $S: H_{p}^{m} \rightarrow \mathbf{R}$, define

$$
\{T, S\}:=\int_{0}^{1} \frac{\delta T}{\delta u} \frac{\partial}{\partial x} \frac{\delta S}{\delta u} d x \quad \text { (Poisson bracket) }
$$

which also is a functional defined on $H_{p}^{m}$. It can be verified that the operation $\{$,$\} has the following properties:$

(i) $\{T, S\}=-\{S, T\}, T, S: H_{p}^{m} \rightarrow \mathbf{R}$;

(ii) $\{H, a T+b S\}=a\{H, T\}+b\{H, S\}, a, b \in R, H, T, S: H_{p}^{m} \rightarrow \mathbf{R}$;

(iii) (Jacobi identity) $\{\{T, S\}, H\}+\{\{S, H\}, T\}+\{\{H, T\}, S\}=0$, $H, T, S: H_{p}^{m} \rightarrow \mathbf{R}$.

Lemma 1. The functional $T(u)$ is a first integral of problem $\left(\mathrm{P}^{\prime}\right)$ if and only if $\{T, H\}=0$.

Proof. Since, for any solution $u=u(t)$ of $\left(\mathrm{P}^{\prime}\right)$,

$$
\frac{d T(u)}{d t}=\int_{0}^{1} \frac{\delta T}{\delta u} \frac{\partial u}{\partial t} d x=\int_{0}^{1} \frac{\delta T}{\delta u} \frac{\partial}{\partial x} \frac{\delta H}{\delta u} d x=\{T, H\}
$$

the lemma follows immediately from this identity.

For a given functional $H: H_{p}^{m} \rightarrow \mathbf{R}$, a family of mappings $G_{H}^{t}$ containing a parameter $t$ can be determined through $\left(\mathbf{P}^{\prime}\right)$ :

$$
u(t)=G_{H}^{t} u_{0}, \quad u_{0} \in H_{p}^{m},
$$

which is called the phase flow corresponding to $H$. By Lemma 1 and the Jacobi 
identity, we have

Theorem 1. Suppose $T$ and $S$ are two first integrals of $\left(\mathrm{P}^{\prime}\right)$. Then $\{T, S\}$ is also a first integral of $\left(\mathbf{P}^{\prime}\right)$. Therefore, the set of functionals consisting of all first integrals of $\left(\mathrm{P}^{\prime}\right)$, equipped with the operation $\{$,$\} , forms a Lie algebra R_{H}$.

Let $L_{u}=-\partial^{2} / \partial x^{2}+u$ (Schrödinger's operator). P. D. Lax proved in [5] that every eigenvalue $\lambda=\lambda(u)$ of the Sturm-Liouville problem $L_{u} f=\lambda f$ is a first integral of $\left(\mathrm{P}^{\prime}\right)$, i.e., $\lambda(u) \in R_{H}$. In fact, $\left(\mathrm{P}^{\prime}\right)$ has infinitely many first integrals, such as

$$
I_{0}(u)=\int_{0}^{1} u d x, I_{1}(u)=\int_{0}^{1} u^{2} d x, I_{2}(u)=\int_{0}^{1}\left(\frac{u_{x}^{2}}{2}+u^{3}\right) d x, \ldots
$$

From the form $\left(\mathbf{P}^{\prime}\right)$ and the properties indicated above we see that problem $(\mathrm{P})$ is of the same nature as a Hamiltonian system of ordinary differential equations (see [1, Chapter 8]), which can be viewed as an infinite-dimensional Hamiltonian system. For a given functional $H: H_{p}^{m} \rightarrow \mathbf{R}$, we call $J \delta H / \delta u$ the velocity vector of the phase flow $G_{H}^{t}$ with Hamiltonian function $H$. For any $I_{s} \in R_{H}$, the phase flow determined by the equation $u_{t}=J \delta I_{s} / \delta u$ commutes with $G_{H}^{t}$, i.e., $G_{H}^{t} G_{I_{s}}^{t}=G_{I_{s}}^{t} G_{H}^{t}$.

\section{The HAMiltonian APPROXimation OF PROBlem (P)}

In this paper we seek to develop a numerical method for simulating the solitary waves of the Korteweg-de Vries equation which maintains the Hamiltonian nature of this equation. We believe that such a method will be able to preserve as much as possible the global properties of the original problem, for example, the energy conservation property

$$
\frac{d H(u)}{d t}=\frac{d}{d t} \int_{0}^{1}\left(\frac{u_{x}^{2}}{2}+u^{3}\right) d x=0
$$

which we consider to be particularly important. As is known, the conventional finite difference method (see [7]) and the Galerkin finite element method (see [8]) do not preserve the energy. In this section, we shall show that the PetrovGalerkin finite element discretization is an appropriate way to derive a numerical method for problem $(P)$ which faithfully preserves the Hamiltonian nature and the energy conservation property of the continuous problem.

Let $L_{h}: 0=x_{0}<x_{1}<\cdots<x_{N}=1$ be a partition of the interval $I=[0,1]$, $I_{j}=\left[x_{j-1}, x_{j}\right]$, and $h=\max _{1 \leq j \leq N}\left(x_{j}-x_{j-1}\right)$. For a given integer $r \geq 2$, we introduce the spaces

$$
\begin{aligned}
& V_{h}=\left\{v \in H_{p}^{1} ;\left.v\right|_{I_{j}} \in P_{r}\left(I_{j}\right), j=1,2, \ldots, N\right\}, \\
& H_{h}=\left\{w \in H_{p}^{2} ;\left.w\right|_{I_{j}} \in P_{r+1}\left(I_{j}\right), j=1,2, \ldots, N\right\},
\end{aligned}
$$

where $P_{r}\left(I_{j}\right)$ represents the set of all polynomials on $I_{j}$ with degree $<r$. It is easy to see that $\operatorname{dim} V_{h}=\operatorname{dim} H_{h}=(r-1) N$. 
Based on the chosen pair of spaces $V_{h}$ and $H_{h}$, the Petrov-Galerkin finite element approximation of problem (P) is defined as follows: find a map $u^{h}(t)$ from $\mathbf{R}^{+}$to $V_{h}$ such that

$$
\left(u_{t}^{h}, w^{h}\right)+3\left(\left(u^{h}\right)^{2}, w_{x}^{h}\right)+\left(u_{x}^{h}, w_{x x}^{h}\right)=0 \quad \forall w^{h} \in H_{h} .
$$

Here and hereafter, $($,$) and \|\cdot\|$ stand for the inner product and the norm in $L_{2}(I)$, respectively.

For the purpose of the subsequent analysis, we introduce a linear integration operator $G: H_{p}^{m} \rightarrow H_{p}^{m+1}$ uniquely determined by

$$
(G f)_{x}=f-f^{0}, \quad(G f)^{0}=f^{0}, \quad f \in H_{p}^{m},
$$

where $f^{0}=(f, 1)$ is the mean value of $f$ on the interval $I$. In fact, $G f$ has the following explicit form:

$$
(G f)(x)=\int_{0}^{x} f(s) d s-f^{0} x+\frac{3}{2} f^{0}-\int_{0}^{1} \int_{0}^{x} f(s) d s d x .
$$

From the definition of $G$, we see that

$$
\begin{gathered}
\left(G f_{1}, f_{2}\right)=\left(G f_{1},\left(G f_{2}\right)_{x}\right)+f_{1}^{0} f_{2}^{0}, \\
(G f, f)=\left(f^{0}\right)^{2} .
\end{gathered}
$$

Moreover, with $\stackrel{\circ}{H}_{p}^{m}=\left\{v \in H_{p}^{m} ; v^{0}=(v, 1)=0\right\}$ and $\stackrel{\circ}{V}_{h}=V_{h} \cap \stackrel{\circ}{H}_{p}^{1}$, we have

$$
\left(G f_{1}, f_{2}\right)=\left(G f_{1},\left(G f_{2}\right)_{x}\right)=-\left(f_{1}, G f_{2}\right) \text { for any } f_{1}, f_{2} \in \stackrel{\circ}{H}_{p}^{m},
$$

i.e., $G$ is a skewsymmetric operator on $\stackrel{\circ}{H}_{p}^{m}$. It can be verified that $G$ is a oneto-one map from $\stackrel{\circ}{H}_{p}^{m}$ onto $\stackrel{\circ}{H}_{p}^{m+1}$, and its inverse is precisely the differential operator $J=\partial / \partial x$.

Theorem 2. The solution $u^{h}=u^{h}(t)$ of the semidiscrete problem $\left(\mathbf{P}_{h}\right)$ satisfies the following conservation laws:

(i) $I_{0}\left(u^{h}(t)\right)=\int_{0}^{1} u^{h} d x=$ const for $t \geq 0$,

(ii) $I_{2}\left(u^{h}(t)\right)=\int_{0}^{1}\left(\frac{\left(u_{x}^{h}\right)^{2}}{2}+\left(u^{h}\right)^{3}\right) d x=$ const for $t \geq 0$.

Proof. Since $1 \in H_{h}$, by choosing $w^{h}=1$ in $\left(\mathbf{P}_{h}\right)$, we have

$$
\frac{d}{d t} \int_{0}^{1} u^{h} d x=\frac{d}{d t}\left(u^{h}, 1\right)=\left(u_{t}^{h}, 1\right)=0,
$$

so that (i) holds. To verify (ii), we choose $w^{h}=G u_{t}^{h} \in H_{h}$; then

$$
\left(u_{t}^{h}, G u_{t}^{h}\right)+3\left(\left(u^{h}\right)^{2},\left(G u_{t}^{h}\right)_{x}\right)+\left(u_{x}^{h},\left(G u_{t}^{h}\right)_{x x}\right)=0 .
$$


Since $\left(u_{t}^{h}\right)^{0}=\left(u_{t}^{h}, 1\right)=0,\left(u_{t}^{h}, G u_{t}^{h}\right)=0$, and $\left(G u_{t}^{h}\right)_{x}=u_{t}^{h}$, because of $(2.1)$ and (2.4), we obtain from the above equation

$$
\frac{d}{d t} I_{2}\left(u^{h}(t)\right)=\frac{d}{d t}\left\{\left(\left(u^{h}\right)^{3}, 1\right)+\frac{1}{2}\left(u_{x}^{h}, u_{x}^{h}\right)\right\}=0,
$$

i.e., (ii) holds, and the theorem is proved.

Theorem 2 tells us that the conservation laws $I_{0}=$ const and $I_{2}=$ const of problem (P) mentioned in $\S 1$ are faithfully preserved by the Petrov-Galerkin finite element approximation $\left(\mathrm{P}_{h}\right)$, where $I_{2}=H$ represents the energy of the continuous system $(\mathrm{P})$.

It is not difficult to see that the discrete problem $\left(\mathbf{P}_{h}\right)$ is a system of ordinary differential equations. After some careful manipulations, we find that $\left(\mathbf{P}_{h}\right)$ is precisely a finite Hamiltonian system. To show this, we introduce a kind of second-order discrete derivative $d_{x x}^{h} u^{h} \in V_{h}$ for any given function $u^{h}$ in $V_{h}$, which is uniquely determined by

$$
\left(d_{x x}^{h} u^{h}, v^{h}\right)=-\left(u_{x}^{h}, v_{x}^{h}\right) \quad \forall v^{h} \in V_{h} .
$$

By choosing $v^{h}=1$, we see that $\left(d_{x x}^{h} u^{h}, 1\right)=0$, i.e., $d_{x x}^{h} u^{h} \in \stackrel{\circ}{V}_{h}=V_{h} \cap \stackrel{\circ}{H}_{p}^{1}$. Now let $u^{h}=u^{h}(t)$ be a solution of problem $\left(\mathbf{P}_{h}\right)$. Since $d_{x x}^{h} u^{h}, u_{t}^{h} \in \stackrel{\circ}{V}_{h}$, by using (2.1) and (2.2), equation $\left(P_{h}\right)$ can be rewritten in the form

$$
\left(G u_{t}^{h}, v^{h}\right)-3\left(\left(u^{h}\right)^{2}, v^{h}\right)+\left(d_{x x}^{h} u^{h}, v^{h}\right)=0, \quad v^{h} \in \stackrel{\circ}{V}_{h} .
$$

In addition, let $P_{0}$ be the $L_{2}$ projector from $L_{2}(I)$ into its subspace $\stackrel{\circ}{V}_{h}$, and let $G_{h}:=P_{0} G$; then for any $f^{h}, g^{h} \in \stackrel{\circ}{V}_{h}$,

$$
\left(G_{h} f^{h}, g^{h}\right)=\left(P_{0} G f^{h}, g^{h}\right)=\left(G f^{h}, g^{h}\right)=-\left(f^{h}, G g^{h}\right)=-\left(f^{h}, G_{h} g^{h}\right),
$$

which shows that $G_{h}$ is a skewsymmetric operator on $\stackrel{\circ}{V}_{h}$. In terms of these notations, we find that $(2.5)$ is equivalent to

$$
G_{h}\left(u^{h}\right)_{t}=3 P_{0}\left(u^{h}\right)^{2}-d_{x x}^{h} u^{h}
$$

It can be verified by calculation that $3 P_{0}\left(u^{h}\right)-d_{x x}^{h} u^{h}=\delta H\left(u^{h}\right) / \delta u^{h}$. Therefore, the solution $u^{h}(t)$ of $\left(\mathrm{P}_{h}\right)$ satisfies

$$
G_{h}\left(u^{h}\right)_{t}=\delta H\left(u^{h}\right) / \delta u^{h}
$$

Assume that $P_{0} \stackrel{\circ}{H}_{h}=\stackrel{\circ}{V}_{h}$; then $G_{h}$ restricted to $\stackrel{\circ}{V}_{h}$ is a one-to-one mapping, and the inverse $G_{h}^{-1}=J_{h}$ exists, which also is a skewsymmetric operator on $\stackrel{\circ}{V}_{h}$. We thus obtain a new version of $\left(\mathrm{P}_{h}\right)$,

$$
\left(u^{h}\right)_{t}=J_{h} \delta H\left(u^{h}\right) / \delta u^{h}
$$


For any two functionals $T, S: V_{h} \rightarrow R$, a discrete analogue of the Poisson bracket, introduced in $\S 1$, can be defined by

$$
\{T, S\}:=\int_{0}^{1} \frac{\delta T}{\delta u^{h}} J_{h} \frac{\delta S}{\delta u^{h}} d x,
$$

and most of the analysis and conclusions in [5] can be carried over to the approximation problem $\left(\mathrm{P}_{h}\right)$. Comparing the form (2.7) of problem $\left(\mathrm{P}_{h}\right)$ with $\left(\mathbf{P}^{\prime}\right)$, we see that the Hamiltonian nature of problem $(\mathbf{P})$ is maintained in the discrete approximation $\left(\mathbf{P}_{h}\right)$. For this reason, we shall call $\left(\mathbf{P}_{h}\right)$ a Hamiltonian approximation of problem $(\mathrm{P})$.

\section{ERROR ESTIMATES AND SUPERCONVERGENCE OF THE APPROXIMATE SOLUTION}

The discrete approximation $\left(\mathrm{P}_{h}\right)$ is identical to one of the methods proposed in [9], where $H^{0}$ and $H^{1}$ estimates for the error $e=u-u^{h}$ and its time derivative $e_{t}$ were derived. However, in the bound obtained for $e_{t}$ there exists an unknown term $\left\|G w_{t}^{h}(0)\right\|_{2}$. In order to achieve superconvergence, D. N. Arnold and R. Winther in [2] altered the discrete equation by a perturbation term. In this section, we obtain superconvergence properties of the unperturbed equation $\left(\mathrm{P}_{h}\right)$ by suitable choices of the initial data.

Since $G\left(H_{p}^{1}\right)=H_{p}^{2}$ and $G\left(V_{h}\right)=H_{h}$, problem $\left(\mathrm{P}_{h}\right)$ can be formulated as follows: find a map $u^{h}(t):[0, T] \rightarrow V_{h}$ such that

$$
-\left(G u_{t}^{h}, v^{h}\right)+3\left(\left(u^{h}\right)^{2}, v^{h}\right)+a_{0}\left(u^{h}, v^{h}\right)=0 \quad \forall v^{h} \in \stackrel{\circ}{V}_{h},
$$

where $a_{0}(u, v)=\left(u_{x}, v_{x}\right)$ and $u^{h}(0)$ assumes a prescribed value in $V_{h}$. In order to be sure that the problem has a unique solution, we assume $P_{0} \stackrel{\circ}{H}_{h}=\stackrel{\circ}{V}_{h}$; then the coefficient matrix in front of the time derivative in (3.1) is nonsingular.

An elliptic projector $P_{1}: H_{p}^{1} \rightarrow V_{h}$ is defined by

$$
\begin{gathered}
a_{0}\left(P_{1} \phi-\phi, v^{h}\right)=0 \quad \text { for any } v^{h} \in V_{h}, \\
\left(P_{1} \phi, 1\right)=(\phi, 1) .
\end{gathered}
$$

Let $u(t)=u(x, t)$ be the exact solution of $(\mathrm{P})$, which is assumed to be sufficiently smooth. From standard results for the Galerkin finite element method for elliptic equations, we know that

$$
\begin{gathered}
\left\|\left(P_{1} u-u\right)^{(k)}(t)\right\|_{s} \leq C(u) h^{r-s}, \quad-(r-2) \leq s \leq 1, \quad k \geq 0, \\
\left\|\left(P_{1} u-u\right)(t)\right\|_{L_{\infty}(I)} \leq C(u) h^{r},
\end{gathered}
$$

where $\phi^{(k)}(t)=\left(\frac{d}{d t}\right)^{k} \phi(t)$. Moreover, the following superconvergence estimate at nodes holds (see [6]):

$$
\left|\left(P_{1} u-u\right)\left(x_{i}, t\right)\right| \leq C(u) h^{2 r-2} \text { when } r>2 .
$$


Here and hereafter, $\|\cdot\|_{s}$ represents the norm in the Sobolev space $H^{s}(I)$, $s \geq 0$, and

$$
\|\cdot\|_{-s}=\sup _{0 \neq v \in H^{s}} \frac{(\cdot, v)}{\|v\|_{s}} .
$$

In the subsequent analysis, we shall use the inverse properties of $\left\{V_{h}\right\}$, such as

$$
\left\|v^{h}\right\|_{1} \leq C h^{-1}\left\|v^{h}\right\| \quad \forall v^{h} \in V_{h} .
$$

It is well known that such properties can be guaranteed by assuming the family $\left\{L_{h}, h>0\right\}$ of partitions to be quasi-uniform, i.e., there is a constant $c>0$ such that $h_{j}=x_{j}-x_{j-1} \geq c h$ for $1 \leq j \leq N$.

To begin with, we discuss the case $u^{h}(0)=P_{1} u(0)$ and prove the following pointwise error estimates.

Theorem 3. Suppose that $(\mathrm{P})$ has a unique solution $u(t)$ for $0 \leq t \leq T, u(t)$ is sufficiently smooth, and $\left\{L_{h}, h>0\right\}$ is quasi-uniform. Assume $u^{h}(0)=P_{1} u(0)$. Then for small $h>0$, the discrete problem $\left(\mathrm{P}_{h}\right)$ has a unique solution $u^{h}(t)$, $0 \leq t \leq T$, which satisfies

$$
\begin{gathered}
\left\|u(t)-u^{h}(t)\right\|_{L_{\infty}(I)} \leq C(u) h^{r}, \\
\left|u\left(x_{i}, t\right)-u^{h}\left(x_{i}, t\right)\right| \leq C(u) h^{r+d}, \quad i=1,2, \ldots, N,
\end{gathered}
$$

where $d=0$ for $r=2$, and $d=1$ for $r>2$.

Proof. Set $z(t)=u(t)-P_{1} u(t)$ and $w^{h}(t)=P_{1} u(t)-u^{h}(t)$. Then $e(t)=$ $u(t)-u^{h}(t)=z(t)+w^{h}(t)$, where $w^{h}(t) \in \stackrel{\circ}{V}_{h}$ satisfies

$$
-\left(G w_{t}^{h}, v^{h}\right)+a_{0}\left(w^{h}, v^{h}\right)=\left(G z_{t}, v^{h}\right)+3\left(\left(u^{h}\right)^{2}-u^{2}, v^{h}\right) \quad \forall v^{h} \in \stackrel{\circ}{V}_{h} .
$$

Since $\left(G w_{t}^{h}, w_{t}^{h}\right)=0$, choosing $v^{h}=w_{t}^{h}$ in (3.7) yields

$$
\frac{1}{2} \frac{d}{d t}\left\|w_{x}^{h}\right\|^{2}=\left(G z_{t}, w_{t}^{h}\right)+3\left(\left(u^{h}\right)^{2}-u^{2}, w_{t}^{h}\right) .
$$

Noting that $\left(u^{h}\right)^{2}-u^{2}=\left(w^{h}\right)^{2}-2\left(P_{1} u\right) w^{h}-\left(P_{1} u+u\right) z$, we have

$$
\begin{aligned}
\frac{1}{2} \frac{d}{d t}\left\|w_{x}^{h}\right\|^{2}= & \frac{d}{d t}\left(G z_{t}, w^{h}\right)-\left(G z_{t t}, w^{h}\right) \\
& +\frac{d}{d t}\left[\left(\left(w^{h}\right)^{3}, 1\right)-3\left(\left(P_{1} u\right) w^{h}, w^{h}\right)-3\left(\left(P_{1} u+u\right) z, w^{h}\right)\right] \\
& +3\left(\left(P_{1} u_{t}\right) w^{h}, w^{h}\right)+3\left(\left(P_{1} u+u\right) z_{t}+\left(P_{1} u_{t}+u_{t}\right) z, w^{h}\right) .
\end{aligned}
$$

Without loss of generality we may assume

$$
\left\|w^{h}(t)\right\|_{1} \leq 1 \text { for } 0 \leq t \leq T .
$$

In fact, this assumption can be removed by the later estimates combined with the inverse inequalities in $V_{h}$ (see [8]). By the smoothness of $u(t)$ and estimate 
(3.2), $\left\|P_{1} u\right\|_{1}$ and $\left\|P_{1} u_{t}\right\|_{1}$ are uniformly bounded for $0<h<h_{0}$ in $0 \leq t \leq$ $T$. Note that $w^{h}(0)=0$ by the choice of $u^{h}(0)$. Integrating (3.8) from 0 to $t$, we obtain in the usual way

$$
\begin{aligned}
\left\|w_{x}^{h}(t)\right\|^{2} \leq C\{ & \|z(t)\|_{-1}^{2}+\left\|z^{(1)}(t)\right\|_{-1}^{2}+\left\|G w^{h}(t)\right\|_{1}^{2} \\
& \quad+\int_{0}^{t}\left[\|z(s)\|_{-1}^{2}+\left\|z^{(1)}(s)\right\|_{-1}^{2}+\left\|z^{(2)}(s)\right\|_{-1}^{2}\right. \\
& \left.\left.+\left\|G w^{h}(s)\right\|_{2}^{2}\right] d s\right\},
\end{aligned}
$$

where $C$ is a constant which does not depend on $h$, but depends on $u$ and its derivatives.

To derive an estimate for $G w^{h}(t)$, we choose $v^{h}=P_{0} G w^{h}$ in (3.7) and obtain

$$
\begin{aligned}
\frac{1}{2} \frac{d}{d t}\left\|P_{0} G w^{h}\right\|^{2} & =a_{0}\left(w^{h}, P_{0} G w^{h}\right)-\left(G z_{t}, P_{0} G w^{h}\right)-3\left(\left(u^{h}\right)^{2}-u^{2}, P_{0} G w^{h}\right) \\
& \leq C\left(\|z\|_{-1}^{2}+\left\|z^{(1)}\right\|_{-1}^{2}+\left\|G w^{h}\right\|_{2}^{2}\right) .
\end{aligned}
$$

Thus, by integration we have

$$
\left\|P_{0} G w^{h}(t)\right\|^{2} \leq C \int_{0}^{t}\left[\|z(s)\|_{-1}^{2}+\left\|z^{(1)}(s)\right\|_{-1}^{2}+\|G w(s)\|_{2}^{2}\right] d s
$$

and

$$
\begin{aligned}
\left\|G w^{h}(t)\right\|^{2} & \leq 2\left\|P_{0} G w^{h}(t)\right\|^{2}+2\left\|\left(I-P_{0}\right) G w^{h}(t)\right\|^{2} \\
& \leq C\left\{h^{4}\left\|G w^{h}(t)\right\|_{2}^{2}+\int_{0}^{t}\left[\|z(s)\|_{-1}^{2}+\left\|z^{(1)}(s)\right\|_{-1}^{2}\right.\right. \\
& \left.\left.+\left\|G w^{h}(s)\right\|_{2}^{2}\right] d s\right\} .
\end{aligned}
$$

Since $\left\|w^{h}\right\|^{2} \leq\left\|G w^{h}\right\|\left\|w_{x}^{h}\right\|+\left\|G w^{h}\right\|^{2}$, combining (3.9) and (3.10) and applying Gronwall's lemma, we find for $h>0$ small enough,

$$
\begin{aligned}
\left\|G w^{h}(t)\right\|_{2}^{2} \leq C\{ & \|z(t)\|_{-1}^{2}+\left\|z^{(1)}(t)\right\|_{-1}^{2} \\
& \left.+\int_{0}^{t}\left[\|z(s)\|_{-1}^{2}+\left\|z^{(1)}(s)\right\|_{-1}^{2}+\left\|z^{(2)}(s)\right\|_{-1}^{2}\right] d s\right\},
\end{aligned}
$$

which shows by (3.2) that

$$
\left\|G w^{h}(t)\right\|_{2} \leq C(u) h^{r+d}
$$

where $d=0$ for $r=2$, and $d=1$ for $r>2$. In view of

$$
\left\|w^{h}(t)\right\|_{L_{\infty}(I)} \leq C\left\|G w^{h}(t)\right\|_{2},
$$

the desired estimates (3.5) and (3.6) can be derived from (3.11) combined with (3.3) and (3.4), respectively. 
From estimate (3.6), we see that the approximate solution has a superconvergence property at the nodes, with one order higher when $r>2$. Following a referee's suggestion, we now improve this result. We shall use the technique of quasi-projection, introduced in [3] for linear second-order parabolic and hyperbolic equations. In [2], quasi-projection was used for the Korteweg-de Vries equation. Since we intend to conserve the energy integral and the Hamiltonian nature, we use this technique only for choosing a suitable initial data, unlike [2], where the discrete equation is altered.

Set $V(t)=P_{1} u(t), Z_{0}(t)=u(t)-V(t)$, and $W_{0}^{h}(t)=V(t)-u^{h}(t)$. The quasi-projections $Z_{j}(t):[0, T] \rightarrow \stackrel{\circ}{V}_{h}, j=1,2, \ldots$, are defined inductively by

$$
a_{0}\left(Z_{j}, v^{h}\right)=\left(G Z_{j-1}^{(1)}-6 u Z_{j-1}, v^{h}\right) \quad \forall v^{h} \in \stackrel{\circ}{V}_{h}, 0 \leq t \leq T .
$$

We shall use the sum $Z_{1}(0)+Z_{2}(0)+\cdots+Z_{m}(0)$ to modify the previous initial data $V(0)=P_{1} u(0)$, i.e., we choose $u^{h}(0)=V(0)-\left[Z_{1}(0)+Z_{2}(0)+\cdots+Z_{m}(0)\right]$, where $m=[(r-1) / 2]$.

The improved superconvergence result is then as follows:

Theorem 4. Assume $(\mathrm{P})$ and $\left\{L_{h}, h>0\right\}$ to be as in Theorem 3 and $u^{h}(0)=$ $V(0)-\left[Z_{1}(0)+Z_{2}(0)+\cdots+Z_{m}(0)\right], m=[(r-1) / 2]$. Then for $h>0$ small enough, the approximate solution $u^{h}(t)$ satisfies

$$
\left|u\left(x_{i}, t\right)-u^{h}\left(x_{i}, t\right)\right| \leq C(u) h^{2 r-2}, \quad i=1,2, \ldots, N .
$$

To illustrate, let $r=4$; then $m=1$ and $u^{h}(0)=V(0)-Z_{1}(0)$. The calculation of $u^{h}(0)$ requires three projections $V(0),\left(Z_{0}\right)_{t}(0)$, and $Z_{1}(0)$, where $\left(Z_{0}\right)_{t}(0)=u_{t}(0)-V_{t}(0)$ and $V_{t}(0)$ is a solution of

$$
a_{0}\left(V_{t}(0), v^{h}\right)=\left(G u_{t t}(0)-6 u(0) u_{t}(0), v^{h}\right), \quad v^{h} \in \stackrel{\circ}{V}_{h} .
$$

The extra cost spent on calculating $V_{t}(0)$ and $Z_{1}(0)$ will be compensated by a convergence rate of order $O\left(h^{6}\right)$.

Now we sketch the proof of Theorem 4.

Let $Z(t)=\sum_{j=0}^{m} Z_{j}(t)$ and $W^{h}(t)=W_{0}^{h}(t)-\sum_{j=1}^{m} Z_{j}(t)$. Then

$$
e(t)=u(t)-u^{h}(t)=Z_{0}(t)+W_{0}^{h}(t)=Z(t)+W^{h}(t),
$$

where $W_{0}^{h}(t), W^{h}(t) \in \stackrel{\circ}{V}_{h}$. It is not difficult to see that $W_{0}^{h}(t)$ and the sum of $Z_{j}(t), j=1,2, \ldots, m$, satisfy respectively the following two equations,

$$
-\left(G\left(W_{0}^{h}\right)^{(1)}-6 u W_{0}^{h}, v^{h}\right)+a_{0}\left(W_{0}^{h}, v^{h}\right)=\left(G Z_{0}^{(1)}-6 u Z_{0}+3 e^{2}, v^{h}\right)
$$

and

$$
\begin{gathered}
-\left(G\left(\sum_{j=1}^{m} Z_{j}\right)^{(1)}-6 u \sum_{j=1}^{m} Z_{j}, v^{h}\right)+a_{0}\left(\sum_{j=1}^{m} Z_{j}, v^{h}\right) \\
=\left(G Z_{0}^{(1)}-6 u Z_{0}, v^{h}\right)-\left(G Z_{m}^{(1)}-6 u Z_{m}, v^{h}\right) .
\end{gathered}
$$


Thus, by subtraction we derive an equation for $W^{h}(t)$,

$$
-\left(G\left(W^{h}\right)^{(1)}-6 u W^{h}, v^{h}\right)+a_{0}\left(W^{h}, v^{h}\right)=\left(G Z_{m}^{(1)}-6 u Z_{m}+3 e^{2}, v^{h}\right) .
$$

By the assumption on $u^{h}(0)$, we have $W^{h}(0)=0$.

The proof of (3.12) consists of estimating $Z(t)$ and $W^{h}(t)$.

Lemma 2. Let $s \geq-1$ and $k, j \geq 0$ be integers such that $2 j+s \leq r-2$. Then

$$
\left|Z_{j}\left(x_{i}, t\right)\right| \leq C(u) h^{2 r-2}, \quad j=1,2, \ldots, m ; i=1,2, \ldots, N .
$$

These estimates may be proved by an argument as in [2] or [3], with some obvious changes.

The next step is to show

$$
\left\|W^{h}(t)\right\|_{1} \leq C(u) h^{2 r-2}, \quad 0 \leq t \leq T .
$$

Then the proof of (3.12) will be completed by (3.4), (3.15), and (3.16). We first choose $v^{h}=\left(W^{h}\right)_{t}$ in (3.13) to obtain

$$
\begin{aligned}
\frac{1}{2} \frac{d}{d t}\left\|W_{x}^{h}\right\|^{2}= & -3 \frac{d}{d t}\left(u W^{h}, W^{h}\right)+3\left(u_{t} W^{h}, W^{h}\right) \\
& +\frac{d}{d t}\left(G Z_{m}^{(1)}-6 u Z_{m}, W^{h}\right) \\
& -\left(G Z_{m}^{(2)}-6 u Z_{m}^{(1)}-6 u_{t} Z_{m}, W^{h}\right)+3\left(e^{2},\left(W^{h}\right)_{t}\right)
\end{aligned}
$$

In addition to (3.17), by choosing $v^{h}=P_{0} G W^{h}$ in (3.13) and integrating this equation from 0 to $t$, we get

$$
\begin{aligned}
& \left\|P_{0} G W^{h}(t)\right\| \\
& \quad \leq C \int_{0}^{t}\left(\left\|W^{h}(s)\right\|_{1}^{2}+\sum_{k=0}^{1}\left\|Z_{m}^{(k)}(s)\right\|_{-1}^{2}+\|e(s)\|^{2}\|e(s)\|_{1}^{2}\right) d s .
\end{aligned}
$$

For lack of available bounds for $\left(W^{h}\right)_{t}$ and $e_{t}$, we treat the nonlinear term $3\left(e^{2},\left(W^{h}\right)_{t}\right)$ of $(3.17)$ in the following way:

$$
\begin{aligned}
3\left(e^{2},\left(W^{h}\right)_{t}\right) & =3\left(Z^{2}+2 Z W^{h}+\left(W^{h}\right)^{2},\left(W^{h}\right)_{t}\right) \\
& =\frac{d}{d t}\left[3\left(Z^{2}, W^{h}\right)+3\left(Z W^{h}, W^{h}\right)+\left(\left(W^{h}\right)^{3}, 1\right)\right] \\
& -6\left(Z Z_{t}, W^{h}\right)-3\left(Z_{t} W^{h}, W^{h}\right) .
\end{aligned}
$$

As in the proof of Theorem 3, we may assume $\left\|W^{h}(t)\right\|_{1} \leq 1,0 \leq t \leq T$; then 
$\left|\left(\left(W^{h}(t)\right)^{3}, 1\right)\right| \leq C\left\|W^{h}(t)\right\|^{2}$. Integrating (3.17), we obtain

$$
\begin{aligned}
\left\|W_{x}^{h}(t)\right\|^{2} \leq C\{ & \left\|W^{h}(t)\right\|^{2}+\sum_{k=0}^{1}\left\|Z_{m}^{(k)}(t)\right\|_{-1}^{2}+\|Z(t)\|^{2}\|Z(t)\|_{1}^{2} \\
+\int_{0}^{t}\left[\left\|W^{h}(s)\right\|_{1}^{2}\right. & +\sum_{k=0}^{2}\left\|Z_{m}^{(k)}(s)\right\|_{-1}^{2} \\
& \left.\left.+\|Z(s)\|^{2}\left\|Z^{(1)}(s)\right\|^{2}\right] d s\right\},
\end{aligned}
$$

where $\left|\left(Z^{(k)} W^{h}, W^{h}\right)\right| \leq C\left\|W^{h}\right\|^{2}, k=0,1$, are implicitly used. Lemma 2 tells us that $\left\|Z_{m}^{(k)}(t)\right\|_{-1} \leq C h^{2 r-2}$ and $\left\|Z^{(k)}(t)\right\|_{s} \leq C h^{r-s}$, for $k=0,1,2$, $s=0,1$, and $0 \leq t \leq T$. Thus, by (3.19),

$$
\left\|W_{x}^{h}(t)\right\|^{2} \leq C\left\{h^{2(2 r-2)}+\left\|W^{h}(t)\right\|^{2}+\int_{0}^{t}\left\|W^{h}(s)\right\|_{1}^{2} d s\right\} .
$$

Since [9] $\|e(t)\|_{s} \leq C h^{r-s}, s=0,1$, and $\left\|\left(I-P_{0}\right) G W^{h}(t)\right\| \leq C h^{2}\left\|W^{h}(t)\right\|_{1}$, we have by $(3.18)$

$$
\left\|G W^{h}(t)\right\|^{2} \leq C\left\{h^{4}\left\|W^{h}(t)\right\|_{1}^{2}+h^{2(2 r-2)}+\int_{0}^{t}\left\|W^{h}(s)\right\|_{1}^{2} d s\right\} .
$$

Similar to the proof of (3.11), when $h>0$ is small enough, the desired estimate (3.16) can be derived from (3.20), (3.21), and Gronwall's lemma. Thus, the proof of Theorem 4 is complete.

\section{NUMERICAL RESULTS OF SIMULATING 1-SOLITARY WAVES}

A numerical experiment is performed for the following solitary wave of $(P)$ with initial data:

$$
\begin{aligned}
& u_{0}(x)=-\left(3 d^{2}\right)^{-1}[1+q(x)], \quad 0 \leq x \leq 1, \\
& q(x)=q_{0}+a \operatorname{sech}^{2}\left(a / 6 d^{2}\right)^{1 / 2}(x-0.5), \\
& q_{0}=-2 d(6 a)^{1 / 2} \tanh \left(a / 24 d^{2}\right)^{1 / 2}
\end{aligned}
$$

where $a=0.2$ and $d=10^{-2}$. Here, $u_{0}(x)$ is extended as a 1-periodic function to the whole real axis, and we denote the corresponding solution of $(P)$ by $u(x, t)$; then $q(x, s)=-1-3 d^{2} u\left(x, \frac{1}{2} d^{2} s\right)$ solves the following equation:

$$
q_{s}+(1+q) q_{x}+\frac{1}{2} d^{2} q_{x x x}=0
$$

The solitary wave $u(x, t)$ is simulated by means of the method $\left(\mathrm{P}_{h}\right)$ with $r=2$ and uniform mesh $x_{j}=j h, h=1 / 47$, while the approximate solution $u^{h}(t)$ is a piecewise linear function. Let $\left\{q_{j}(x) ; j=1,2, \ldots, 47\right\}$ be the basis of the subspace $V_{h}$, and

$$
u^{h}(x, t)=\sum_{j=1}^{47} u_{j}(t) q_{j}(x)
$$


Then it can be seen that $\left\{u_{j}(t) ; j=1,2, \ldots, 47\right\}$ is the solution of the system of ordinary differential equations

$$
\begin{gathered}
\sum_{j=1}^{47} a_{i j} \frac{d u_{j}}{d t}-\frac{1}{h^{3}}\left(u_{i-1}-2 u_{i}+u_{i+1}\right)+\frac{1}{4 h}\left(u_{i-1}^{2}+6 u_{i}^{2}+u_{i+1}^{2}\right) \\
+\frac{1}{2 h}\left(u_{i-1} u_{i}+u_{i} u_{i+1}\right)-\sum_{j=1}^{47}\left(u_{j}^{2}+u_{j} u_{j+1}+u_{j+1}^{2}\right)=0
\end{gathered}
$$

where $a_{i j}=\left(q_{j}, G q_{i}\right) / h^{2}$, and by the periodicity, $u_{0}=u_{47}, u_{1}=u_{48}$.

We choose the time step $\Delta t=3.125 \times 10^{-7}$ and discretize (4.1) in the time variable by the midpoint rule; then a fully discrete scheme for $(\mathrm{P})$ is obtained, namely

$$
\begin{aligned}
\sum_{j=1}^{47} a_{i j} \frac{u_{j}^{n+1}-u_{j}^{n}}{\Delta t}=F_{i}\left(\frac{u^{n+1}+u^{n}}{2}\right), \quad i=1,2, \ldots, 47 \\
n=0,1, \ldots,
\end{aligned}
$$

where

$$
\begin{aligned}
F_{i}(v)= & \frac{1}{h^{3}}\left(v_{i-1}-2 v_{i}+v_{i+1}\right)-\frac{1}{4 h}\left(v_{i-1}^{2}+6 v_{i}^{2}+v_{i+1}^{2}\right) \\
& -\frac{1}{2 h}\left(v_{i-1} v_{i}+v_{i} v_{i+1}\right)+\sum_{j=1}^{47}\left(v_{j}^{2}+v_{j} v_{j+1}+v_{j+1}^{2}\right) .
\end{aligned}
$$

As pointed out by Feng Kang in [4], the midpoint rule (i.e., the centered implicit Euler scheme) is a symplectic scheme, which behaves very well as far as preserving conservation laws is concerned.

Table 1 indicates the ability of scheme (4.2) to preserve the conservation laws $I_{i}=$ const , $i=0,1,2$, when this scheme is used to simulate the solitary waves of $(\mathrm{P})$.

Figures 1-3 exhibit the shapes of solitary waves $q(x, s)$ calculated by scheme (4.2) at time steps $n=0,30,60$, respectively.

\section{TABLE 1}

Values of $I_{i}, i=0,1,2$, at various time steps

\begin{tabular}{|c|r|r|c|}
\hline$n$ & $I_{0}(u)$ & $I_{1}(u)$ & $I_{2}(u)$ \\
\hline 0 & -3333.33333 & 11137605.2 & $-373082079 \times 10^{2}$ \\
\hline 30 & -3333.33333 & 11137605.0 & $-373082041 \times 10^{2}$ \\
\hline 60 & -3333.33448 & 11137624.0 & $-373082365 \times 10^{2}$ \\
\hline 90 & -3333.33206 & 11138221.0 & $-373081466 \times 10^{2}$ \\
\hline 140 & -3333.33251 & 11138159.6 & $-373081527 \times 10^{2}$ \\
\hline 190 & -3333.33141 & 11138299.3 & $-373081693 \times 10^{2}$ \\
\hline
\end{tabular}




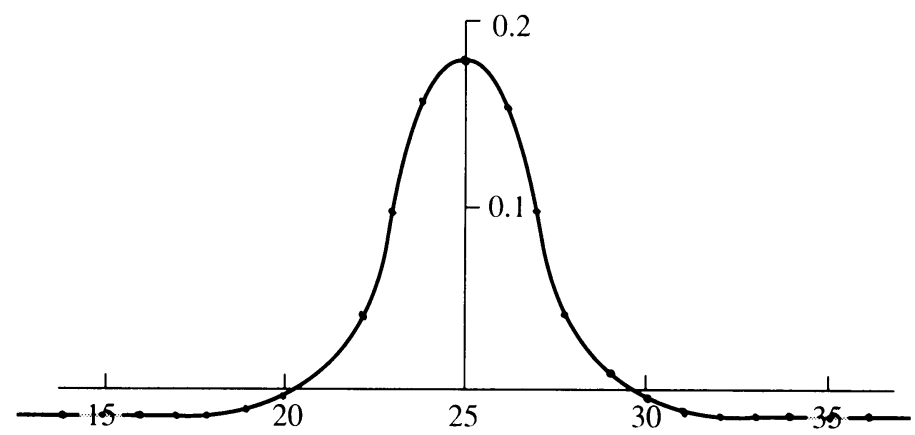

FIGURE 1

The shape of solitary wave $q(x, s)$ at time step $n=0$

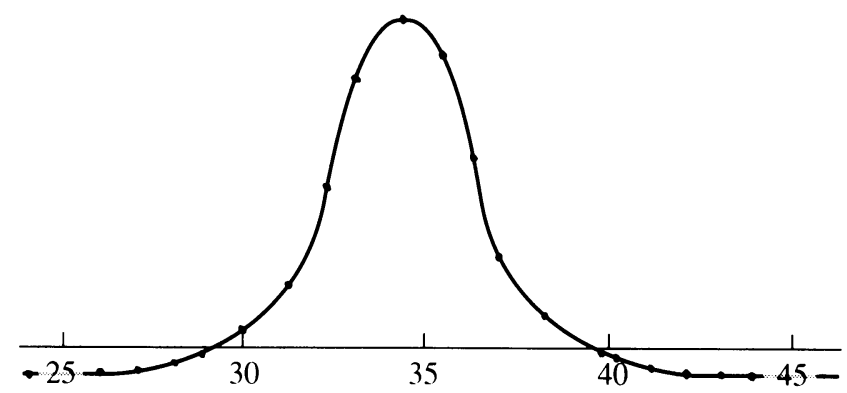

FIGURE 2

The shape of solitary wave $q(x, s)$ at time step $n=30$

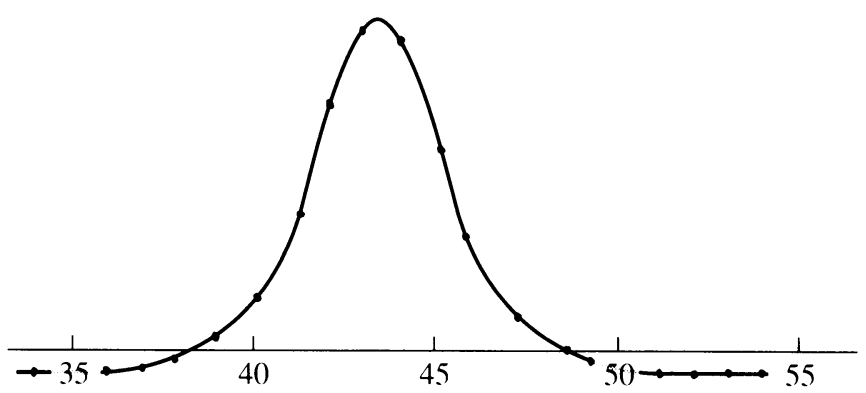

Figure 3

The shape of solitary wave $q(x, s)$ at time step $n=60$

\section{BIBLIOGRAPHY}

1. V. I. Arnold, Mathematical methods of classical mechanics, Springer-Verlag, 1978.

2. D. N. Arnold and R. Winther, A superconvergent finite element method for the Korteweg-de Vries equation, Math. Comp. 38 (1982), 23-36.

3. J. Douglas, Jr., T. Dupont, and M. F. Wheeler, A quasi-projection analysis of Galerkin methods for parabolic and hyperbolic equations, Math. Comp. 32 (1978), 345-362. 
4. Feng Kang, On difference schemes and symplectic geometry, Proc. 1984 Beijing Symposium on Differential Geometry and Differential Equations (Feng Kang, ed.), Science Press, Beijing, 1985, pp. 42-58.

5. P. D. Lax, Integrals of nonlinear equations of evolution and solitary waves, Comm. Pure Appl. Math. 21 (1968), 467-490.

6. V. Thomée, Negative norm estimates and superconvergence in Galerkin methods for parabolic problems, Math. Comp. 34 (1980), 93-113.

7. A. C. Vliegenthart, On finite difference methods for the Korteweg-de Vries equation, J. Engrg. Math. 5 (1971), 137-155.

8. L. B. Wahlbin, A dissipative Galerkin method for the numerical solution of first order hyperbolic equations, Mathematical Aspects of Finite Element Methods in P.D.E.s (C. de Boor, ed.), 1974.

9. R. Winther, A conservative finite element method for the Korteweg-de Vries equation, Math. Comp. 34 (1980), 23-43.

Department of Mathematics, Jilin University, Changchun 130023, People's Republic OF CHINA 